\title{
Factors Affecting the Implementation of Early Rehabilitation Care in Patients with Traumatic Brain Injury: A Multidisciplinary Perspective
}

\author{
Mohammad Eghbali $^{1,2}$, Hamidreza Khankeh ${ }^{3,4} *$ (D) Sayed Ali Hosseini ${ }^{5}$, Abbas Ebadi ${ }^{6,7}$, Vafa Rahimi-Movaghar ${ }^{8}$ \\ Received: 1 Feb 2021 \\ Published: 4 Nov 2021
}

\begin{abstract}
Background: Traumatic brain injury is now regarded as the silent epidemic. This disease can cause some physical, cognitive, and psychological impairments that require proper and early rehabilitation interventions. Therefore, the aim of this study was to explore the factors affecting early rehabilitation care in patients with traumatic brain injury based on the experiences of the rehabilitation team.

Methods: This qualitative study was conducted as a conventional content analysis from September 2019 to August 2020. For this purpose and given the maximum diversity, 22 members of the rehabilitation team, patients, and their caregivers in trauma level 1 hospitals affiliated with Tehran University of Medical Sciences were selected based on a purposeful sampling method. The data were also collected using semi-structured interviews until data saturation is obtained. Graneheim and Lundman's content analysis method was performed to analyze the data.

Results: The data analysis results eventually led to the introduction of three main themes and eight categories. The main themes were associated with early rehabilitation barriers, which included cultural factors and rehabilitation infrastructures. Rehabilitation management was regarded as the main theme regarding the early rehabilitation facilitator.

Conclusion: Early rehabilitation is considered a vital rehabilitation stage for patients with traumatic brain injury. Hence, accurate identification of the influential factors on early rehabilitation can help the rehabilitation team promote early rehabilitation care among
\end{abstract} these patients; it can lead to the revival of the patients' abilities and the improvement of their quality of life.

Keywords: Traumatic Brain Injury, Early Rehabilitation, Qualitative Research, Content Analysis

Conflicts of Interest: None declared

Funding: This article is extracted from a Ph.D. dissertation submitted to the University of Social Welfare and Rehabilitation Siciences through the following code: ir.uswr.rec.1396.215. The project was conducted with the financial help of the research deputy of the University of Social Welfare and Rehabilitation Sciences.

\section{*This work has been published under CC BY-NC-SA 1.0 license.}

Copyright $₫$ Iran University of Medical Sciences

Cite this article as: Eghbali M, Khankeh $\mathrm{H}$, Hosseini SA, Ebadi A, Rahimi-Movaghar V. Factors Affecting the Implementation of Early Rehabilitation Care in Patients with Traumatic Brain Injury: A Multidisciplinary Perspective. Med J Islam Repub Iran. 2021 (4 Nov);35:147. https://doi.org/10.47176/mjiri.35.147

\section{Introduction}

Traumatic brain injury (TBI) is mainly caused by a blunt and penetrated trauma or acceleration/deceleration force that occurs to the brain and its tissues (1). This damage can lead to the reduction of the level of consciousness,
Corresponding author: Dr Hamidreza Khankeh, hamid.khankeh@ki.se

1. Department of Nursing University of Social Welfare and Rehabilitation Sciences, Tehran, Iran

2. Torbat Heydariyeh University of Medical Sciences, Torbat Heydariyeh, Iran

3. Emergency and Disaster Health Research Center, University of Social Welfare and Rehabilitation Sciences, Tehran, Iran

4. Department of Clinical Science and Education, Karolinska Institute, Stockholm, Sweden

5. Social Determinants of Health Research Center, Occupational Therapy Department, University of Social Welfare and Rehabilitation Sciences, Tehran, Iran

6. Behavioral Sciences Research Center, Life Style Institute, Baqiyatallah University of Medical Sciences, Tehran, Iran

7. Faculty of Nursing, Baqiyatallah University of Medical Sciences, Teheran, Iran

8. Sina Trauma and Surgery Research Center, Tehran University of Medical Sciences, Tehran, Iran $\uparrow$ What is "already known" in this topic:

Early rehabilitation is an integral part of the rehabilitation process which is considered as the prerequisite of other treatment stages for patients with TBI. Early rehabilitation interventions can lead to functional outcomes among the patients with TBI and may reduce the hospital stay, particularly in the ICU, for such patients.

\section{$\rightarrow$ What this article adds:}

Management of rehabilitation, as a facilitator factor, includes specialized team care and rehabilitation planning. However, cultural factors (attitudes to rehabilitation) and rehabilitation infrastructure (lack of resources) are among the barriers to rehabilitation interventions. The findings can also contribute to the improvement of quality of life, physical problems, and mental health. 
memory loss or forgetfulness, neurological problems, or even death $(2,3)$.

TBI is regarded as an important public health issue as well as a socio-economic problem worldwide. The incidences of TBI are increasing annually (3). It is reported that about 2.8 million people suffer from TBI in the United States each year (4). The direct and indirect costs of brain injuries are estimated to be between $\$ 60.4$ billion and \$ 221 billion in the US (5). TBI is considered as the second leading cause of death in Iran given that road traffic accidents are the main cause; $48 \%$ of the accidents have occurred to motorcyclists within the age range of 21 to 40 years (6).

Given the advances in medical care services, the mortality rates, as well as the number of surviving patients with severe brain injuries, have increased over the past few decades. However, the forthcoming sensory-motor and neurological problems have created challenges for these patients and rehabilitation medicine (7). Although the majority of the patients with TBI recover within several weeks or months following the injury, it is reported that more than 3 million Americans should live with disabilities that are caused by brain injury $(5,8)$.

TBI can result in permanent or temporary functional disorders in the brain that may lead to limited patient activities, affect community participation, and reduce the quality of life. These problems can lead to depression and other chronic illnesses among these people $(9,10)$. Patients with brain injuries require long-term but costly care, including intensive care services and rehabilitation treatments (11).

Modern medical sciences are dealing with the rehabilitation of patients after TBI (12). Therefore, early, integrated, and appropriate rehabilitation care is vital to revive and improve brain function as well as the individual's return to society (13). Early rehabilitation interventions are crucial for the following stages of the patients' rehabilitation care. Providing early, interdisciplinary, and specialized rehabilitation can help improve patients' participation in such activities, regain performance, and enhance the patients' quality of life (14). Family is regarded as one of the main members of the rehabilitation team for patients with TBI; given the economic advantage and also the therapeutic benefits of family involvement, it is considered crucial in the patients' rehabilitation process (15). Moreover, the active participation of the family members in rehabilitation can lead to the improvement of the patient's recovery rate (16).

According to the results of the previous studies, early rehabilitation care can reduce the patients' hospital stay, especially in the intensive care unit. It can also result in improved functioning outcomes, regaining of individual's independence, and thus reduction of the treatment costs $(17,18)$. In these studies, it was recommended to further conduct qualitative or mixed-method studies in order to investigate the barriers and facilitators of early rehabilitation activities in the intensive care unit (17).

Despite the benefits of early rehabilitation, more than half of the patients admitted to intensive care units do not receive such services. Besides, the majority of the patients
$(>95 \%)$ remained unmoved within the first 72 hours after hospitalization, and the rate of implementation of early rehabilitation care is low $(19,20)$. Several studies have examined the barriers and facilitators of early mobilization for the patients admitted to the intensive care unit (19-22); however, there is no comprehensive study in the literature examining the factors affecting early rehabilitation in patients with traumatic brain injury from the perspective of the members of the rehabilitation team. Therefore, the present study was conducted to investigate and describe the factors affecting the implementation of early rehabilitation care among patients with TBI in order to create a clear image for the more efficient provision of these services in Iran.

\section{Methods}

\section{Study design}

This qualitative study was conducted using the conventional content analysis approach from September 2019 to August 2020. Qualitative content analysis is used as a systematic method when the researchers aim to provide a regular classification of the collected data as well as to discover the explicit and hidden themes or patterns in the data. Regarding conventional content analysis, the researchers avoid using pre-determined categories and seek new categories based on the data $(23,24)$.

\section{Setting}

The participants in this study included different members of the rehabilitation team and also the patients and their caregivers who were seliected from Sina and Imam Khomeini Hospitals in Tehran, Iran, as well as prehospital emergency wards. It is noteworthy that the two target hospitals are regarded as medical centers for patients with trauma. The researcher should refer to different hospital wards such as intensive care units, neurology wards, and emergency wards so as to identify the members of the specialized rehabilitation team for the patients with TBI as well as their caregivers who are considered as a rich source of information.

\section{Study Participants and Sampling}

In this study, purposeful sampling was used given the maximum diversity in terms of age, sex, work experience, specialty, and level of education. The following inclusion criteria were taken into account for patients and their caregivers as a part of the rehabilitation team: 1) age of over 18 years, 2) traumatic brain injury, 3) mild to severe injury based on the Glasgow Coma Scale (where the GCS of 13-15 refers to mild, GCS of 9-12 refers to moderate, and GCS of 3-8 refers to severe TBI), and 4) willingness and ability to participate in the study. In addition, the following inclusion criteria were applied for the members of the professional rehabilitation team: 1) at least two years of experience in the related specialized field, 2) the ability to communicate appropriately and based on a rich experience, as well as 3) having enough time to participate in the interviews and share their experiences. 


\section{Data collection}

The data were collected through in-depth and semistructured individual interviews tailored to the research question. The data collection process began in September 2019 and lasted until August 2020. A total number of 23 interviews were conducted with 22 members of the rehabilitation team, patients, and their caregivers. Initially, the time and the place of the interviews were specified after face-to-face coordination with each of the participants. To ensure the convenience and privacy of the interviewees, the participants were asked to select the appropriate place and time for the interviews. The interview guideline questions were prepared for the interviews (Table 1). The interviews would continue until data saturation was achieved and the categories were completed. All the interviews were conducted by the first author of the article (ME) who was well-trained regarding qualitative studies and interviews. The interviews were recorded upon the participants' consent and were then transcribed word by word into the Microsoft Word software. The interview process was also evaluated and guided by other authors who were experts in qualitative research.

\section{Data analysis}

The conventional content analysis method was used to analyze the data according to the steps which were proposed by Graneheim and Lundman (25). In this method, each of the recorded interviews would be transcribed word by word, and the interviews were reviewed several times meticulously. The transcribed interviews were initially reviewed as a whole in order for the researchers to be acquainted with the texts. The actual analysis began with looking for the semantic units which were related to different aspects of barriers and facilitators of early rehabilitation. These units were then condensed, abstracted, and then labeled, provided that their original meaning is retained. Given the similarities and differences, the codes were used to form the subcategories. Finally, the subcategories were re-read and then further categorized based on the similarities and their relationship with the other subcategories. Eventually, the main theme was extracted. Preliminary analyses were performed by the leading researcher (ME) and were then evaluated and finalized by the other authors. Since the researchers did not observe any new code after conducting the twenty-three interviews, it can be confirmed that data saturation has been achieved. MAXQDA version 10 was used for data analysis.

\section{Trustworthiness and Rigor}

In this study, four criteria of credibility, dependability, transferability, and confirmability - which were proposed by Guba and Lincoln $(26,27)$ - were used to validate the data. The credibility of the study was established through the prolonged engagement of the participants and the setting for about one year, dedication of enough time to collect the data, member-checking, peer-debriefing, and simultaneous analysis by the researcher in order to confirm the validity and acceptability of the data. Moreover, it was intended to involve the majority of the rehabilitation team and particularly the patients and their families in the data collection procedure (source triangulation). The research process and different stages of the study were recorded and reported in detail and thorough audit trail notes as well as logs in order to comply with dependability. In order to highlight the transferability of the study findings, the researchers provided a comprehensive and meticulous description and analysis of the research setting as well as a detailed explanation of the participants and the context. They also presented a clear and rich description of the effective factors on early rehabilitation so as to inform fellow researchers and the readers about the research procedures in order to be implemented in different social contexts. In order to express the confirmability of this research, the findings and the trials regarding the interviews, the codes, and the extracted categories were reviewed and confirmed by the authors (peer-debriefing), two respected faculty members, as well as a rehabilitation care specialist.

\section{Ethical Consideration}

This study is a part of the Ph.D. dissertation that was evaluated and approved by the ethics committee of the University of Social Welfare and Rehabilitation Sciences (no.IR.USWR.REC.1396.215). The participants were informed of the purpose of the study, and then their written informed consent was obtainedl. The participants were also ensured that they could leave the study at any time. They also agreed to participate voluntarily in the present study and they were assured that all the data were anonymous.

\section{Results}

\section{Characteristics of Study Participants}

The majority of the participants in this study were male (13), and the rest were female (9). The demographic information indicated an average age range of $40.45 \pm 7.68$ years and a mean work experience of 15.41 years for the

Table 1. Interview guide

\begin{tabular}{ll}
\hline Multidisciplinary team & Question \\
\hline Patient & What has happened since the injury? \\
& What services have you received after the injury? \\
& Who (Which groups) provided related services to you after the injury? \\
& How do you evaluate those services? \\
Rehabilitation Team & What is your treatment approach for patients with TBI? \\
& What types of services are provided to the patients with TBI? What services do you provide? \\
& What rehabilitation services are provided to the patients with TBI right after admission to the hospital? and How? \\
& Are these services useful (based on your experience)? \\
& What specialties are included in the early stages of rehabilitation interventions? \\
& Please express your experiences regarding providing early rehabilitation care to the patients with TBI. \\
& In your opinion, what equipment and what measures are necessary for more efficient delivery of such services?
\end{tabular}




\begin{tabular}{lcc}
\hline \multicolumn{2}{l}{ Table 2. Participant characteristics $(\mathrm{N}=22)$} & \\
\hline Variable & Level & $\mathrm{n}(\%)$ \\
\hline Gender & Male & $13(59.1 \%)$ \\
& Female & $9(40.9 \%)$ \\
Marital status & Single & $10(45 \%)$ \\
Rehabilitation Team & Married & $12(55 \%)$ \\
& Nurse & $7(31.8 \%)$ \\
& Patient and caregiver & $5(22.8 \%)$ \\
& Physician & $5(22.8 \%)$ \\
Education Status & PT/OT* & $3(13.6 \%)$ \\
& Logopedist & $1(4.5 \%)$ \\
& Psychologist & $1(4.5 \%)$ \\
Years of experience & Diploma & $2(9.1 \%)$ \\
& Bachelor & $8(36.3 \%)$ \\
Duration of interview & Master & $5(22.8 \%)$ \\
(min) & PhD & $7(31.8 \%)$ \\
*PT: physical therapist, OT: occupational therapist & $5-29$ \\
\end{tabular}

participants. Besides, the interview lasted for 32.11 minutes on average (Table 2). Finally, 271 codes were extracted as a result of the data analysis, which was then classified into 3 main themes and 8 subcategories.

\section{Factors affecting early rehabilitation}

The main themes included rehabilitation management, cultural factors, and rehabilitation infrastructures which are shown in Table 3.

Rehabilitation Management: Based on the findings of the interviews, rehabilitation management was regarded as one of the main facilitating factors in early rehabilitation among patients with traumatic brain injury. It refers to the existence of some specific conditions and factors in this process. This main category includes two subcategories, i.e. rehabilitation planning and specialized team care.

Rehabilitation planning: The participants in this study stated that there is a need for planning in the first stages of interventions in order to provide proper rehabilitation care. Then, given the comprehensive evaluation of the patients and their capabilities, a specialized and capable team should be used to perform such interventions. In this regard, one of the specialists in rehabilitation and physical medicine with ten years of work experience asserted that:

"These patients must be evaluated by a professional rehabilitation team. The ICF model of the patients' problems should be examined through the comprehensive evaluation of the patients." (P 17, rehabilitation medicine)

Specialized rehabilitation services are considered as one of the special needs of these patients in order to experience a quick recovery. In this regard, one of the neurologists stated that:

"In fact, if the patients receive more comprehensive and more specialized measures and facilities, it will yield better results for the patients. In other words, the occupational therapists and physiotherapists should be welltrained in these areas in order to be able to provide the optimal rehabilitation services to the patient." (P 6, neurologist)

In addition to the comprehensive evaluation of the nerve system, it is necessary to conduct an analysis of the patients' nutritional demands. In this regard, one of the experienced nurses argued:

"We need nutritional consultation because we know that the patients with TBI are in a state of hypercatabolism; i.e., they show high metabolism indexes. However, such evaluations must be conducted by physical therapists or nutritionists. The nutrition status and the micronutrients that they receive should be assessed by a specialist using the necessary tests." (P 16, nurse)

Specialized Team Care: Early rehabilitation care for patients with TBI requires a capable and multidisciplinary team that can examine the patient from different perspectives and can cooperate closely in this regard. This team can consist of a physiotherapist, occupational therapist,

Table 3. Factors affecting the implementation of early rehabilitation care in TBI

\begin{tabular}{|c|c|c|}
\hline Theme & Categories & Subcateg,ory \\
\hline Rehabilitation Management & $\begin{array}{l}\text { Rehabilitation Planning } \\
\text { Specialized Team Care }\end{array}$ & $\begin{array}{l}\text { Primary Comprehensive Evaluation } \\
\text { Planning } \\
\text { Specialized care as the pre-requisite for improvement } \\
\text { Teamwork } \\
\text { Capable Multidisciplinary Team } \\
\text { Specialized Team Perspective } \\
\text { Importance of Awareness of the Patient and Their Families }\end{array}$ \\
\hline Cultural Factors & $\begin{array}{l}\text { Detrimental Cultural Factors } \\
\text { Attitude toward Rehabilitation }\end{array}$ & $\begin{array}{l}\text { Helping Culture } \\
\text { Benefactors Helping Culture } \\
\text { Dominant Therapeutic Culture } \\
\text { Early Outcomes } \\
\text { Long-Term Rehabilitation } \\
\text { Adherence to Rehabilitation }\end{array}$ \\
\hline Rehabilitation Infrastructure & $\begin{array}{l}\text { Structural-Policy Problems } \\
\text { Lack of Resources } \\
\text { Lack of Knowledge/Attitude }\end{array}$ & $\begin{array}{l}\text { Lack of Rehabilitation Hospitals } \\
\text { Dominant Policies in the Ministry of Health } \\
\text { Lack of Rehabilitation Wards } \\
\text { Limited Insurance Coverage for Rehabilitation } \\
\text { Limited Budget for Rehabilitation } \\
\text { Insufficient Equipment } \\
\text { Lack of Workforce } \\
\text { Families' lack of Knowledge } \\
\text { Treatment Team's Lack of Knowledge } \\
\text { Physicians' low Willingness }\end{array}$ \\
\hline
\end{tabular}


nurse, physical medicine and rehabilitation specialist, neurosurgeon, speech therapist, ICU specialist, psychologist, as well as the patients' family members. If this specialized team care is provided to the patients in a timely manner, it will lead to quick recovery and early discharge from the hospital. In this case, one of the participants stated that:

"The treatment of these patients is team-based. We work together. For example, sometimes the nurses may not notice some issues or I might not be aware of some procedures. Then, we would help each other and act as the complements." (P 5, physiotherapist)

The rehabilitation team does not only include professional and specialized members but also involves the patients, their family members, or the caregivers. In fact, the patients and their families should be taught about the rehabilitation interventions, treatment procedures, and preparing the family for further care and rehabilitation. Hence, one of the nurses stated that:

"The patients who are planned to be discharged by tracheostomy need to receive the required instructions by the nurses and the anesthesiologists in the hospital wards." (P 16 , nurse)

\section{Cultural factors}

Cultural factors are regarded as one of the effective barriers against early rehabilitation. In fact, these cultural factors can influence the whole process of early rehabilitation care. Detrimental cultural factors and attitudes toward rehabilitation are the two main elements of cultural factors.

Detrimental Cultural Factors: The helping culture at the time of the accident sometimes leads to irreparable injuries to the patients. On the other hand, the dominant therapeutic culture (lack of belief in rehabilitation services, delays in rehabilitation care, \& the traditional view toward rehabilitation) prevents the proper implementation of rehabilitation services in these patients. Benefactors are also less willing to invest in the field of rehabilitation. In this regard, one of the participants asserted that:

"The most important thing is to quit the traditional atmosphere, i.e. to avoid the stereotypes such as we conducted the surgery and then it is what it is or the patients experienced a brain injury so we should not bother them anymore. They mainly believed that they should stop the treatment process for the patient, although that person could become be an effective person for the society in future." (P 5, occupational therapist)

The dominant culture in the hospital has developed a less important view toward rehabilitation interventions. Accordingly, one of the participants argued that:

"They consider physiotherapy as an unimportant intervention; we have observed it a lot." (P 21, physiotherapist)

Attitude toward Rehabilitation: Patients and their families' attitudes toward rehabilitation are crucial for the implementation of early rehabilitation. The patients and their families should know that rehabilitation is a long-term process and requires constant follow-up interventions. On the other hand, the outcomes of rehabilitation interventions may not be noticeably quick, and patients should not be disappointed accordingly. In this regard, one of the nurses in the intensive care unit stated that:

"It is important for the families to be aware of the longterm nature of rehabilitation interventions. The intervention team began the rehabilitation process, but they need the support and involvement of the families in order to continue with the interventions and achieve the ultimate goals." (P 4, nurse)

Rehabilitation infrastructure: Rehabilitation infrastructure is considered as one of the most important barriers against early rehabilitation in patients with traumatic brain injury. The hospitals' policies mainly focus on treatment, and therefore, it has led to poor rehabilitation infrastructure. Structural-policy problems, the lack of knowledge/attitude, and the lack of resources and environmental conditions are the subcategories of rehabilitation infrastructure.

Structural-Policy Problems": The dominant policies have given lower priorities to rehabilitation interventions compared to therapeutic interventions. Therefore, there are only a limited number of rehabilitation hospitals in Iran; besides, there are almost no rehabilitation wards in general hospitals as well. In this regard, one of the neurologists stated that:

"In fact, there are no rehabilitation departments in our country. In other words, when patients with brain strokes or patients with TBI are discharged from the hospital, there is no Neurological rehabilitation center to refer. Meanwhile, such patients refer" to Neurological rehabilitation wards right after discharge in many countries."(P20, neurologists)

"I think that Ministry of Health should set policies towards rehabilitation and that rehabilitation should be considered as a treatment neciessity along with other disciplines of other specialties". (P17, rehabilitation medicine).

Lack of Resources: Limited financial support regarding rehabilitation and inadequate insurance coverage can result in poorly provided early rehabilitation care or costly services for the families. It is also noteworthy that in addition to sufficient and skilled manpower, it is necessary to supply appropriate and efficient equipment in order to provide better rehabilitation services. The head-nurse in the intensive care unit argued that:

"The biggest challenges are the limited budget of the system as well as the large number of patients referring to the hospitals which makes it difficult to provide efficient services to these patients. It is more difficult for some specific services such as speech therapy and occupational therapy. "(P3, nurse)

In addition to the limited hospital resources to conduct rehabilitation interventions, there is limited insurance coverage in this regard. Thus, one of the participants stated that:

"First of all, rehabilitation is a very expensive field, i.e. the patients do not generally choose this field because of its high costs and its absolutely poor insurance coverage. They just partly cover the physiotherapy services." (P17, rehabilitation medicine)

Lack of Knowledge-Attitude.: It is believed that the patients' family members' limited knowledge about rehabili- 
tation prevents appropriate and early rehabilitation interventions. On the other hand, physicians' also do not have enough knowledge about rehabilitation and its interventions. Besides, since rehabilitation services can generate low income, the physicians are not motivated enough to enter this field. In this regard, one of the rehabilitation medicine experts stated that:

"Unfortunately, the students or the graduates do not acquire sufficient knowledge in the university to enter this field. They are really experts in the treatment of pain or regarding skeletal-muscular problems, and they can manage these conditions very well; however, I'm afraid this is not true in rehabilitation services." (P10, rehabilitation medicine)

Accordingly, one of the occupational therapists also asserted that: "I hope that our surgical community in various fields of surgery such as neurosurgery and orthopedics, which we are especially dealing with a lot, as well as the neurologists have a rehabilitation perspective toward treatment. In other words, they should not only consider the patients from medical perspectives. They should believe that the patients need other types of treatments as well, and I think all of them should accept and believe that a rehabilitation team can be regarded as a great help for their patients." (P 5, occupational therapist)

\section{Discussion}

This study investigated and identified the factors affecting early rehabilitation cares in patients with TBI. The results of the study showed that rehabilitation management includes rehabilitation planning and specialized team care, which is regarded as the facilitating factor. On the other hand, cultural factors and rehabilitation infrastructures were identified as the barriers against early rehabilitation interventions in patients with traumatic brain injury.

Rehabilitation planning and specialized team care are important factors that facilitate early rehabilitation care in patients with traumatic brain injury. Eghbali et al. conducted a study on the analysis of the concept of early rehabilitation in patients with TBI. They concluded that early specialized care and team planning are among the most important components of early rehabilitation interventions (13). In another seminal study, Goddard also found that early rehabilitation is a complex intervention and requires the proper use of different specialties to be performed (20). Rehabilitation involves comprehensive planning regarding hospital care and providing instructions to the patients and their caregivers. In this regard, the results of another study by Downing et al. showed that providing the necessary information about injuries and treatment programs, as well as a good discharge plan whose proper implementation is necessary for rehabilitation (28).

The dominant therapeutic culture, including lack of belief in rehabilitation services and the traditional view toward rehabilitation, has prevented the proper implementation of rehabilitation services in patients with traumatic brain injury. Accordingly, Burke et al. examined the importance and status of rehabilitation; they argued that given the politicians' incomplete and improper knowledge and awareness in this regard, rehabilitation services are of low importance and status for the governments in the world. This, in turn, leads to poor cooperation and, as a result, the provision of inadequate rehabilitation services for patients with TBI (29).

Rehabilitation is a long-term process that requires continuous patient participation and constant interventions. The patients' and their families' attitudes towards rehabilitation and their adherence to such interventions are regarded as the main factors that can delay the provision of the interventions. In addition, Khoshbakht et al. (2019) conducted a study on the factors affecting rehabilitation adherence in patients with brain stroke. They concluded that patient-related factors such as trust in the effectiveness of rehabilitation and family support could play an important role in the patients' adherence to the treatment interventions (30). These findings are in line with the results of the present study due to the similar structure of the study and the target patients.

Rehabilitation infrastructure is considered one of the most important and effective barriers to early rehabilitation care. The dominant policies imposed by the Ministry of Health have led to insufficient resources in the field of rehabilitation, which has led to the shortage of rehabilitation hospitals in Iran. On the other hand, insufficient allocation of the budget has led to a decrease in the specialized workforce as well as an insufficient supply of equipment. Barber et al. stated that lack of resources and facilities is regarded as one of the barriers to early rehabilitation in patients admitted to intensive care units (19). In another seminal study, Goddard et al. examined the barriers and facilitators of early rehabilitation in patients using a ventilator and asserted that the lack of medical staff and the lack of adequate equipment are the main barriers to early rehabilitation (20). In addition, Gupta et al. also concluded that shortage in medical staff (such as nurses, physiotherapists, \& occupational therapists), equipment (such as CT scan devices), financial support, as well as the roles of the family and community have led to delayed delivery of such services. Consequently, it has caused complications for patients with TBI in India (31).

Unfortunately, the high costs of rehabilitation interventions, as well as the limited insurance coverage for these services, have created an excessive financial burden on the patients and their families. In this regard, Khoshbakht conducted a study on the adherence to rehabilitation in patients with stroke in Iran; they found that inadequate insurance coverage with regards to rehabilitation services is considered as one of the important and influential factors on rehabilitation adherence. On the other hand, the rehabilitation team's lack of knowledge and awareness of rehabilitation can also affect the implementation of early rehabilitation in patients with traumatic brain injury. In this regard, Abdi et al. stated that it is necessary to provide various necessary instructions (e.g., knowledge, attitude, \& skills) to rehabilitation service providers. Moreover, they argued that these pieces of training should be examined before graduation (32). Finally, the results of a systematic review study conducted by Mlenzana et al. showed that the members of the treatment team had lim- 
ited knowledge of rehabilitation and also the rehabilitation process (21).

\section{Strengths and limitations}

One of the strengths of this study was the analysis of the factors affecting early rehabilitation care in patients with TBI from a multidisciplinary perspective. The researchers also investigated early rehabilitation based on the opinions of people with a good history of rehabilitation. Besides, interviewing was used as the main method for data collection in this study. Therefore, it is recommended to use other means of data collection (such as focus groups \& observation) in future studies in order to achieve more accurate results. It is also suggested to further investigate the factors which affect the other phases of rehabilitation in such patients.

\section{Conclusion}

The findings of the present study provided the very first evidence regarding the factors affecting early rehabilitation care in patients with traumatic brain injury; such evidence was obtained from the perspective of the rehabilitation team as well as the patients and their caregivers. Since early rehabilitation is the first step in the rehabilitation process for patients with traumatic brain injury, identification of the related facilitators and barriers can lead to a more efficient provision of such interventions and thus better outcomes in patients. The findings of this study identified the following types of barriers: rehabilitation infrastructure (structural-policy problems, lack of resources, \& lack of knowledge/attitude) as well as cultural factors (detrimental cultural factors \& attitudes toward rehabilitation). On the other hand, rehabilitation management, as the facilitating factor, includes rehabilitation planning and specialized team care. Focusing on the facilitators and barriers can lead to better provision of early rehabilitation in these patients, improvement of quality of life, as well as improvement of physical problems and mental health

\section{Acknowledgment}

The researchers express their sincere appreciation and gratitude to all participants who participated in this study.

\section{Conflict of Interests}

The authors declare that they have no competing interests.

\section{References}

1. Eghbali M, Khankeh H, Ebadi A. The importance of early rehabilitation in traumatic brain injury. Nurs Pract Today. $2020 \mathrm{Apr}$ $1 ; 7(2): 84-6$

2. Dang B, Chen W, He W, Chen G. Rehabilitation Treatment and Progress of Traumatic Brain Injury Dysfunction. Neural Plast. 2017:1582182.

3. Van Praag DL, Cnossen MC, Polinder S, Wilson L, Maas AI. Posttraumatic stress disorder after civilian traumatic brain injury: A systematic review and meta-analysis of prevalence rates. J Neurotrauma. 2019 Dec 1;36(23):3220-32.

4.Kreitzer N, Rath K, Kurowski BG, Bakas T, Hart K, Lindsell CJ, et al. Rehabilitation practices in patients with moderate and severe traumatic brain injury. J Head Trauma Rehabil. 2019;34(5):E66-E72.
5.Iaccarino MA, Bhatnagar S, Zafonte R. Rehabilitation after traumatic brain injury. Handbook of Clinical Neurology. 2015;127:411-22.

6.Eslami N, Nourali Z, Ghorbani M, Tabatabaei TSJ, Tabatabaei SSJ. Effectiveness of Cognitive Rehabillitation Group Therapy on Verbal Memory, Stress Management and Alexithymia in Patients with Traumatic Brain Injury. Iran Rehabil J. 2020;6(3):9-16.

7. Jacobsson L, Lexell J. Functioning and disability from 10 to 16 years after traumatic brain injury. Acta Neurol Scand. 2020 Feb;141(2):11522.

8.Griffin AD, Turtzo LC, Parikh GY, Tolpygo A, Lodato Z, Moses AD, et al. Traumatic microbleeds suggest vascular injury and predict disability in traumatic brain injury. Brain. 2019;142(11):3550-64.

9. Mausbach BT, Chattillion EA, Moore RC, Roepke SK, Depp CA, Roesch S. Activity restriction and depression in medical patients and their caregivers: a meta-analysis. Clin Psychol Rev. 2011 Aug 1;31(6):900-8.

10.Froutan R, Eghbali M, Hoseini SH, Mazloom SR, Yekaninejad MS, Boostani R. The effect of music therapy on physiological parameters of patients with traumatic brain injury: A triple-blind randomized controlled clinical trial. Complement Ther Clin Pract. 2020;40:101216.

11. Steiner E, Murg-Argeny M, Steltzer H. The severe traumatic brain injury in Austria: early rehabilitative treatment and outcome. J Trauma Manag Outcomes. 2016;10(1):1-6.

12. Chantsoulis M, Mirski A, Rasmus A, Kropotov JuD, Pachalska M. Neuropsychological rehabilitation for traumatic brain injury patients. Ann Agric Environ Med. 2015;22(2):368-379.

13. Eghbali M, Khankeh H, Hosseinii SA, Rahimi-Movaghar V, Ebadi A. Early Rehabilitation Care for Patients with Traumatic Brain Injury: A Concept Analysis Study. Trauma Mon. 2020;25(5):212-219.

14.Buhl I, Pallesen H. Early rehabilitation of patients with severe acquired brain injury: Strategies to promote participation. Scand J Occup Ther. 2015;22(3):181-95.

15.Fisher A, Bellon M, Lawn S, Lennon S, Sohlberg M. Family-directed approach to brain injury (FAB) model: a preliminary framework to guide family-directed intervention for individuals with brain injury. Disabil Rehabil. 2019;41(7):854-60.

16. Foster AM, Armstrong J, Buckley A, Sherry J, Young T, Foliaki S, James-Hohaia TM, Theadom A, McPherson KM. Encouraging family engagement in the rehabilitation process: A rehabilitation provider's development of support strategies fior family members of people with traumatic brain injury. Disabil Rehabil. 2012 Nov 1;34(22):1855-62.

17.Sosnowski K, Lin F, Mitchell ML, White H. Early rehabilitation in the intensive care unit: an integrative literature review. Aust Crit Care 2015;28(4):216-25.

18.Lui SK, Fook-Chong SMC, Teo QQ. Demographics of traumatic brain injury and outcomes of continuous chain of early rehabilitation in Singapore. Proc Singapore Healthc. 2020;29(1):33-41.

19.Barber EA, Everard T, Holland AE, Tipping C, Bradley SJ, Hodgson CL. Barriers and facilitators to early mobilisation in intensive care: a qualitative study. Aust Crit Care. 2015;28(4):177-82.

20.Goddard SL, Lorencatto F, Koo E, Rose L, Fan E, Kho ME, et al Barriers and facilitators to early rehabilitation in mechanically ventilated patients - a theory-driven interview study. J Intensive Care. 2018;6(1):4.

21. Mlenzana NB, Frantz JM, Rhoda AJ, Eide AH. Barriers to and facilitators of rehabilitation services for people with physical disabilities: A systematic review. Affr J Disabil. 2013;2(1).

22.Hashem MD, Nelliot A, Needham DM. Early mobilization and rehabilitation in the ICU: moving back to the future. Respir Care. 2016;61(7):971-9.

23.Vaismoradi M, Turunen H, Bondas T. Content analysis and thematic analysis: Implications for conducting a qualitative descriptive study. Nurs Health Sci. 2013;15(3):398-405.

24. Graneheim UH, Lindgren BM, Lundman B. Methodological challenges in qualitative content analysis: A discussion paper. Nurse Educ Today. 2017 Sep 1;56:29-34.

25. Renz SM, Carrington JM, Badger TA. Two strategies for qualitative content analysis: An intramethod approach to triangulation. Qual Health Res. 2018 Apr;28(5):824-31.

26.Guba EG. Criteria for assessing the trustworthiness of naturalistic inquiries. Educ Technol Res Dev. 1981;29(2):75.

27.Lincoln YS. Emerging criteria for quality in qualitative and interpretive research. Qual Inq. 1995;1(3):275-89.

28.Downing M, Hicks A, Braaf S, Myles D, Gabbe B, Cameron P, et al. 
Factors facilitating recovery following severe traumatic brain injury: A qualitative study. Neuropsychol Rehabil. 2020:1-25.

29.Burke S, McGettrick G, Foley K, Manikandan M, Barry S. The neuro-rehabilitation implementation framework in Ireland: Challenges for implementation and the implications for people with brain injuries. Health Policy. 2020;124(3):225-30.

30.Pishkhani MK, Dalvandi A, Ebadi A, Hosseini M. Factors affecting adherence to rehabilitation in Iranian stroke patients: A qualitative study. J Vasc Nurs. 2019;37(4):264-71.

31.Gupta S, Khajanchi M, Solomon H, Raykar NP, Alkire BC, Roy N, et al. Traumatic brain injury in Mumbai: A survey of providers along the care continuum. Asian J Neurosurg. 2020;15(3):627.

32.Abdi K, Arab M, Rashidian A, Kamali M, Khankeh HR, Farahani FK. Exploring barriers of the health system to rehabilitation services for people with disabilities in Iran: A qualitative study. Electron. Physician. 2018;7(7):1476. 